\title{
Bernstein Collocation Method for Solving Nonlinear Fredholm-Volterra Integrodifferential Equations in the Most General Form
}

\author{
Ayşegül Akyüz-Daşcıŏlu, ${ }^{1}$ Neşe İşler Acar, ${ }^{2}$ and Coşkun Güler ${ }^{3}$ \\ ${ }^{1}$ Department of Mathematics, Faculty of Arts \& Sciences, Pamukkale University, 20070 Denizli, Turkey \\ ${ }^{2}$ Department of Mathematics, Faculty of Arts \& Sciences, Mehmet Akif Ersoy University, 15030 Burdur, Turkey \\ ${ }^{3}$ Department of Mathematical Engineering, Faculty of Chemistry and Metallurgy, Yildiz Technical University, 34210 Istanbul, Turkey
}

Correspondence should be addressed to Coşkun Güler; cguler@yildiz.edu.tr

Received 14 May 2014; Accepted 17 July 2014; Published 10 August 2014

Academic Editor: Naseer Shahzad

Copyright (C) 2014 Ayşegül Akyüz-Daşcioğlu et al. This is an open access article distributed under the Creative Commons Attribution License, which permits unrestricted use, distribution, and reproduction in any medium, provided the original work is properly cited.

\begin{abstract}
A collocation method based on the Bernstein polynomials defined on the interval $[a, b]$ is developed for approximate solutions of the Fredholm-Volterra integrodifferential equation (FVIDE) in the most general form. This method is reduced to linear FVIDE via the collocation points and quasilinearization technique. Some numerical examples are also given to demonstrate the applicability, accuracy, and efficiency of the proposed method.
\end{abstract}

\section{Introduction}

The quasilinearization method was introduced by Bellman and Kalaba [1] to solve nonlinear ordinary or partial differential equations as a generalization of the Newton-Raphson method. The origin of this method lies in the theory of dynamic programming. In this method, the nonlinear equations are expressed as a sequence of linear equations and these equations are solved recursively. The main advantage of this method is that it converges monotonically and quadratically to the exact solution of the original equations [2]. Therefore, the quasilinearization method is an effective approach for obtaining approximate solutions of nonlinear equations such as differential equations [3-7], functional equations [8, 9], integral equations [10-12], and integrodifferential equations [13-15].

In this paper, we consider the nonlinear FVIDE in the general form

$$
\begin{aligned}
g\left(x, y(x), y^{\prime}(x), \ldots, y^{(m)}(x)\right) \\
=\lambda_{1} \int_{a}^{b} f\left(x, t, y(t), y^{\prime}(t), \ldots, y^{(m)}(t)\right) d t \\
\quad+\lambda_{2} \int_{a}^{x} v\left(x, t, y(t), y^{\prime}(t), \ldots, y^{(m)}(t)\right) d t,
\end{aligned}
$$

under the initial

$$
\sum_{k=0}^{m-1} \tau_{j k} y^{(k)}(c)=\mu_{j} ; \quad j=0,1, \ldots, m-1, c \in[a, b],
$$

or boundary conditions

$$
\sum_{k=0}^{m-1}\left[\alpha_{j k} y^{(k)}(a)+\beta_{j k} y^{(k)}(b)\right]=\gamma_{j} ; \quad j=0,1, \ldots, m-1
$$

Here $g:[a, b] \times \mathbb{R}^{m+1} \rightarrow \mathbb{R}, f:[a, b] \times[a, b] \times \mathbb{R}^{m+1} \rightarrow \mathbb{R}$, and $v:[a, b] \times[a, b] \times \mathbb{R}^{m+1} \rightarrow \mathbb{R}$ are known functions, $\alpha_{j k}$, $\beta_{j k}, \tau_{j k}, \mu_{j}, \gamma_{j}, \lambda_{1}$, and $\lambda_{2}$ are known constants, and $y(x)$ is an unknown function.

Besides, we approximate to the nonlinear FVIDE (1) by the generalized Bernstein polynomials defined on the interval $[a, b]$ as

$$
y(x) \cong B_{n}(y ; x)=\sum_{i=0}^{n} y\left(a+\frac{(b-a) i}{n}\right) p_{i, n}(x),
$$


where $p_{i, n}(x)$ denotes the generalized Bernstein basis polynomials of the form

$$
p_{i, n}(x)=\frac{1}{(b-a)^{n}}\left(\begin{array}{c}
n \\
i
\end{array}\right)(x-a)^{i}(b-x)^{n-i} ; \quad i=0,1, \ldots, n .
$$

For convenience, we set $p_{i, n}(x)=0$, if $i<0$ or $i>n$.

Bernstein polynomials have many useful properties such as the positivity, continuity, differentiability, integrability, recursion's relation, symmetry, and unity partition of the basis set over the interval $[a, b]$. For more information about the Bernstein polynomials, see $[16,17]$. Recently, these polynomials have been used for the numerical solutions of differential equations $[4,18,19]$, integral equations [20-24], and integrodifferential equations [25-27].

Now, we give two main theorems for the generalized Berntein polynomials and their basis forms that were proved by Akyuz Dascioglu and Isler [4] as follows.

Theorem 1. If $y \in C^{k}[a, b]$, for some integer $m \geq 0$, then

$$
\lim _{n \rightarrow \infty} B_{n}^{(k)}(y ; x)=y^{(k)}(x) ; \quad k=0,1, \ldots, m
$$

converges uniformly.

Proof. The above theorem can be easily proved by applying transformation $t=(x-a) /(b-a)$ to the theorem given on the interval $[0,1]$ by Phillips [28].

Theorem 2 (see [4]). There is a relation between generalized Bernstein basis polynomials matrix and their derivatives in the form

$$
\mathbf{P}^{(k)}(x)=\mathbf{P}(x) \mathbf{N}^{k} ; \quad k=1, \ldots, m
$$

such that

$$
\mathbf{P}(x)=\left[\begin{array}{llll}
p_{0, n}(x) & p_{1, n}(x) & \cdots & p_{n, n}(x)
\end{array}\right] .
$$

Here the elements of $(n+1) \times(n+1)$ matrix $\mathbf{N}=\left(d_{i j}\right), i, j=$ $0,1, \ldots, n$, are defined by

$$
d_{i j}=\frac{1}{b-a} \begin{cases}n-i ; & \text { if } j=i+1 \\ 2 i-n ; & \text { if } j=i \\ -i ; & \text { if } j=i-1 \\ 0 ; & \text { otherwise. }\end{cases}
$$

In highlight of these theorems, a collocation method based on the generalized Bernstein polynomials, given in Section 2, is developed for the approximate solutions of the nonlinear FVIDE in the most general form (1) via the quasilinearization technique iteratively. In Section 3, some numerical examples are presented for exhibiting the accuracy and applicability of the proposed method. Finally, the paper ends with the conclusions in Section 4.

\section{Method of the Solution}

Our aim is to obtain a numerical solution of the nonlinear FVIDE in the general form (1) under conditions (2) or (3) in terms of the generalized Bernstein polynomials. For this, we firstly express this nonlinear equation as a sequence of linear FVIDEs via the quasilinearization technique iteratively. After that, using the collocation points yields the system of linear algebraic equations. This system represents a matrix equation given by the following theorem. Finally, solving this system with the conditions we get the desired approximate solution.

Theorem 3. Let $x_{s}$ be collocation points defined on the interval $[a, b]$, and let the functions $g, f$, and $v$ be able to expand by Taylor series with respect to $y^{(k)} ; k=0,1, \ldots, m$. Suppose that nonlinear FVIDE (1) has the generalized Bernstein polynomial solution. Then, the following matrix relation holds:

$$
\begin{array}{r}
{\left[\sum_{k=0}^{m}\left(\mathbf{G}_{r, k} \mathbf{P}-\lambda_{1} \mathbf{F}_{r, k}-\lambda_{2} \mathbf{V}_{r, k}\right) \mathbf{N}^{k}\right] \mathbf{Y}_{r+1}=\mathbf{H}_{r} ;} \\
r=0,1, \ldots,
\end{array}
$$

where $\mathbf{N}$ is defined in Theorem 2, $\mathbf{P}=\left[p_{i, n}\left(x_{s}\right)\right], \mathbf{G}_{r, k}=$ $\operatorname{diag}\left[G_{r, k}\left(x_{s}\right)\right], \mathbf{F}_{r, k}=\left[F_{s, i}^{r, k}\right]$, and $\mathbf{V}_{r, k}=\left[V_{s, i}^{r, k}\right]$ are $(n+1) \times$ $(n+1)$ matrices, and $\mathbf{Y}_{r+1}=\left[y_{r+1}(a+(b-a) i / n)\right]$ and $\mathbf{H}_{r}=\left[h_{r}\left(x_{s}\right)\right]$ are $(n+1) \times 1$ matrices for $i, s=0,1, \ldots, n$, such that

$$
\begin{gathered}
G_{r, k}\left(x_{s}\right)=g_{y_{r}^{(k)}}\left(x_{s}, y_{r}\left(x_{s}\right), y_{r}^{\prime}\left(x_{s}\right), \ldots, y_{r}^{(m)}\left(x_{s}\right)\right), \\
F_{s, i}^{r, k}=\int_{a}^{b} f_{y_{r}^{(k)}}\left(x_{s}, t, y_{r}(t), y_{r}^{\prime}(t), \ldots, y_{r}^{(m)}(t)\right) p_{i, n}(t) d t \\
V_{s, i}^{r, k}=\int_{a}^{x_{s}} v_{y_{r}^{(k)}}\left(x_{s}, t, y_{r}(t), y_{r}^{\prime}(t), \ldots, y_{r}^{(m)}(t)\right) p_{i, n}(t) d t .
\end{gathered}
$$

Here $r$ is iteration index, $p_{i, n}(x)$ is generalized Bernstein basis polynomials, and $h_{r}(x)$ is given in the following proof.

Proof. Firstly, by applying the quasilinearization method to the nonlinear FVIDE (1), we obtain a sequence of linear FVIDEs:

$$
\begin{aligned}
& g\left(x, y_{r}(x), y_{r}^{\prime}(x), \ldots, y_{r}^{(m)}(x)\right) \\
& +\sum_{k=0}^{m}\left(y_{r+1}^{(k)}(x)-y_{r}^{(k)}(x)\right) g_{y_{r}^{(k)}} \\
& =\sum_{k=0}^{m}\left[\lambda_{1} \int_{a}^{b}\left(y_{r+1}^{(k)}(t)-y_{r}^{(k)}(t)\right) f_{y_{r}^{(k)}} d t\right. \\
& \left.\quad+\lambda_{2} \int_{a}^{x}\left(y_{r+1}^{(k)}(t)-y_{r}^{(k)}(t)\right) v_{y_{r}^{(k)}} d t\right] \\
& +\lambda_{1} \int_{a}^{b} f\left(x, t, y_{r}(t), y_{r}^{\prime}(t), \ldots, y_{r}^{(m)}(t)\right) d t \\
& +\lambda_{2} \int_{a}^{x} v\left(x, t, y_{r}(t), y_{r}^{\prime}(t), \ldots, y_{r}^{(m)}(t)\right) d t
\end{aligned}
$$


where the expressions $g_{y_{r}^{(k)}}, f_{y_{r}^{(k)}}$, and $v_{y_{r}^{(k)}}$ represent partial differentiation of the functions $g, f$, and $v$ with respect to $y_{r}^{(k)}$, and these are defined, respectively, as

$$
\begin{aligned}
g_{y_{r}^{(k)}} & =\frac{\partial g}{\partial y_{r}^{(k)}}\left(x, y_{r}(x), y_{r}^{\prime}(x), \ldots, y_{r}^{(m)}(x)\right), \\
f_{y_{r}^{(k)}} & =\frac{\partial f}{\partial y_{r}^{(k)}}\left(x, t, y_{r}(t), y_{r}^{\prime}(t), \ldots, y_{r}^{(m)}(t)\right), \\
v_{y_{r}^{(k)}} & =\frac{\partial v}{\partial y_{r}^{(k)}}\left(x, t, y_{r}(t), y_{r}^{\prime}(t), \ldots, y_{r}^{(m)}(t)\right) .
\end{aligned}
$$

denoting $h_{r}(x)$ :

$$
\begin{aligned}
h_{r}(x)= & -g\left(x, y_{r}, y_{r}^{\prime}, \ldots, y_{r}^{(m)}\right) \\
& +\sum_{k=0}^{m}\left[g_{y_{r}^{(k)}} y_{r}^{(k)}(x)-\lambda_{1} \int_{a}^{b} f_{y_{r}^{(k)}} y_{r}^{(k)}(t) d t\right. \\
& \left.\quad \lambda_{2} \int_{a}^{x} v_{y_{r}^{(k)}} y_{r}^{(k)}(t) d t\right] \\
& +\lambda_{1} \int_{a}^{b} f\left(x, t, y_{r}(t), y_{r}^{\prime}(t), \ldots, y_{r}^{(m)}(t)\right) d t \\
& +\lambda_{2} \int_{a}^{x} v\left(x, t, y_{r}(t), y_{r}^{\prime}(t), \ldots, y_{r}^{(m)}(t)\right) d t .
\end{aligned}
$$

Notice that (14) is a linear FVIDE with variable coefficients, since $y_{r}(x)$ is known function of $x . y_{r+1}(x)$ is an unknown function that has the Bernstein polynomial solution; also this function and its derivatives can be expressed by

$$
y_{r+1}^{(k)}(x) \simeq B_{n}^{(k)}\left(y_{r+1} ; x\right)=\mathbf{P}^{(k)}(x) \mathbf{Y}_{r+1} ; \quad r=0,1, \ldots
$$

By utilizing Theorem 2 and collocation points, above relation becomes

$$
y_{r+1}^{(k)}\left(x_{s}\right)=\mathbf{P}\left(x_{s}\right) \mathbf{N}^{k} \mathbf{Y}_{r+1} ; \quad k=0,1, \ldots, m
$$

Substituting the collocation points and the relation (17) into (14), we obtain a linear algebraic system:

$$
\begin{aligned}
& \sum_{k=0}^{m}\left[G_{r, k}\left(x_{s}\right) \mathbf{P}\left(x_{s}\right)-\lambda_{1} \mathbf{F}_{r, k}\left(x_{s}\right)-\lambda_{2} \mathbf{V}_{r, k}\left(x_{s}\right)\right] \mathbf{N}^{k} \mathbf{Y}_{r+1} \\
& =h_{r}\left(x_{s}\right) .
\end{aligned}
$$

Here $\mathbf{F}_{r, k}\left(x_{s}\right)$ and $\mathbf{V}_{r, k}\left(x_{s}\right)$ are denoted by

$$
\begin{aligned}
\mathbf{F}_{r, k}\left(x_{s}\right) & =\int_{a}^{b} f_{y_{r}^{(k)}}\left(x_{s}, t, y_{r}(t), y_{r}^{\prime}(t), \ldots, y_{r}^{(m)}(t)\right) \mathbf{P}(t) d t \\
\mathbf{V}_{r, k}\left(x_{s}\right) & =\int_{a}^{x_{s}} v_{y_{r}^{(k)}}\left(x_{s}, t, y_{r}(t), y_{r}^{\prime}(t), \ldots, y_{r}^{(m)}(t)\right) \mathbf{P}(t) d t .
\end{aligned}
$$

$\mathbf{P}(t)$ and $G_{r, k}(x)$ are defined, respectively, in Theorems 2 and 3.

For $s=0,1, \ldots, n$, the system (18) can be written compactly in the matrix form

$$
\mathbf{W}_{r} \mathbf{Y}_{r+1}=\mathbf{H}_{r} ; \quad r=0,1, \ldots
$$

so that

$$
\mathbf{W}_{r}=\sum_{k=0}^{m}\left(\mathbf{G}_{r, k} \mathbf{P}-\lambda_{1} \mathbf{F}_{r, k}-\lambda_{2} \mathbf{V}_{r, k}\right) \mathbf{N}^{k}
$$

where matrices are clearly

$$
\begin{gathered}
\mathbf{G}_{r, k}=\left[\begin{array}{cccc}
G_{r, k}\left(x_{0}\right) & 0 & \ldots & 0 \\
0 & G_{r, k}\left(x_{1}\right) & \ldots & 0 \\
\vdots & \vdots & \ddots & \vdots \\
0 & 0 & \ldots & G_{r, k}\left(x_{n}\right)
\end{array}\right], \\
\mathbf{P}=\left[\begin{array}{c}
\mathbf{P}\left(x_{0}\right) \\
\mathbf{P}\left(x_{1}\right) \\
\vdots \\
\mathbf{P}\left(x_{n}\right)
\end{array}\right], \\
\mathbf{V}_{r, k}=\left[\begin{array}{c}
\mathbf{F}_{r, k}\left(x_{0}\right) \\
\mathbf{F}_{r, k}\left(x_{1}\right) \\
\vdots \\
\mathbf{V}_{r, k}\left(x_{0}\right) \\
\mathbf{V}_{r, k}\left(x_{1}\right) \\
\vdots \\
\mathbf{V}_{r, k}\left(x_{n}\right)
\end{array}\right], \\
\mathbf{H}_{r}=\left[\begin{array}{c}
h_{r}\left(x_{0}\right) \\
h_{r}\left(x_{1}\right) \\
\vdots \\
h_{r}\left(x_{n}\right)
\end{array}\right] .
\end{gathered}
$$

Hence, the proof is completed.

Corollary 4. For $\lambda_{1}=\lambda_{2}=0$, the nonlinear FVIDE (1) is reduced to the $m$ th order nonlinear differential equation

$$
g\left(x, y(x), y^{\prime}(x), \ldots, y^{(m)}(x)\right)=0,
$$

and by utilizing Theorem 3, this equation can be written as

$$
\sum_{k=0}^{m} \mathbf{G}_{r, k} \mathbf{P N}^{k} \mathbf{Y}_{r+1}=\widehat{\mathbf{H}}_{r} ; \quad r=0,1, \ldots
$$

Here the matrices $\mathbf{P}, \mathbf{N}$, and $\mathbf{G}_{r, k}$ are defined as above, and elements of the matrix $\widehat{\mathbf{H}}_{r}=\left[\widehat{h}_{r}\left(x_{s}\right)\right]$ are denoted by

$$
\begin{aligned}
\widehat{h}_{r}\left(x_{s}\right)=\sum_{k=0}^{m} G_{r, k}\left(x_{s}\right) y_{r}^{(k)}\left(x_{s}\right) & \\
& \quad-g\left(x_{s}, y_{r}\left(x_{s}\right), y_{r}^{\prime}\left(x_{s}\right), \ldots, y_{r}^{(m)}\left(x_{s}\right)\right) .
\end{aligned}
$$


Corollary 5. For $m=0$, the nonlinear FVIDE (1) is reduced to the nonlinear Fredholm-Volterra integral equation in the form

$$
g(x, y(x))=\lambda_{1} \int_{a}^{b} f(x, t, y(t)) d t+\lambda_{2} \int_{a}^{x} v(x, t, y(t)) d t
$$

such that $y^{(0)}(t)=y(t)$. From Theorem 3, this equation has the iteration matrix form as

$$
\left[\mathbf{G}_{r}-\lambda_{1} \mathbf{F}_{r}-\lambda_{2} \mathbf{V}_{r}\right] \mathbf{Y}_{r+1}=\mathbf{H}_{r} ; \quad r=0,1, \ldots
$$

Here $\mathbf{G}_{r}=\operatorname{diag}\left[G_{r}\left(x_{s}\right)\right], \mathbf{F}_{r}=\left[F_{r, s, i}\right], \mathbf{V}_{r}=\left[V_{r, s, i}\right], \mathbf{H}_{r}=$ $\left[h_{r}\left(x_{s}\right)\right]$, and elements of these matrices are denoted as follows:

$$
\begin{gathered}
G_{r}\left(x_{s}\right)=g_{y_{r}}\left(x_{s}, y_{r}\left(x_{s}\right)\right), \\
F_{r, s, i}=\int_{a}^{b} f_{y_{r}}\left(x_{s}, t, y_{r}(t)\right) p_{i, n}(t) d t, \\
V_{r, s, i}=\int_{a}^{x_{s}} v_{y_{r}}\left(x_{s}, t, y_{r}(t)\right) p_{i, n}(t) d t,
\end{gathered}
$$

$$
\begin{aligned}
& h_{r}\left(x_{s}\right) \\
& =g_{y_{r}}\left(x_{s}, y_{r}\left(x_{s}\right)\right) y_{r}\left(x_{s}\right)-g\left(x_{s}, y_{r}\left(x_{s}\right)\right) \\
& \quad-\lambda_{1} \int_{a}^{b}\left[f\left(x_{s}, t, y_{r}(t)\right)-f_{y_{r}}\left(x_{s}, t, y_{r}(t)\right) y_{r}(t)\right] d t \\
& \quad-\lambda_{2} \int_{a}^{x_{s}}\left[v\left(x_{s}, t, y_{r}(t)\right)-v_{y_{r}}\left(x_{s}, t, y_{r}(t)\right) y_{r}(t)\right] d t .
\end{aligned}
$$

Now we can solve the nonlinear FVDIE (1) under the initial (2) or boundary (3) conditions as follows.

Step 1. Firstly, we use Theorem 3 for the nonlinear FVDIE (1) and determine the matrices in (10). This matrix equation is a system of linear algebraic equations with $n$-unknown coefficients $y_{r+1}(a+(b-a) i / n)$. Let the augmented matrix corresponding equation (10) be denoted by $\left[\mathbf{W}_{r} ; \mathbf{H}_{r}\right]$.

Step 2. We need to choose the first iteration function $y_{0}(x)$ for calculating the $\mathbf{W}_{r}$ and $\mathbf{H}_{r}$. Notice that this function can be obtained in a variety of ways. For instance, it can be obtained from the physical situation for engineering problems. However, a very rough choice for the first iteration function such as initial value is enough for the procedure to converge. We can also consider that the first iteration function as the highest degree polynomial satisfied the given conditions (2) or (3).

Step 3. From expression (17), initial (2) and boundary (3) conditions can be written in the matrix forms, respectively,

$$
\begin{aligned}
& \mathbf{I}_{j} \mathbf{Y}_{r+1}=\mu_{j}, \\
& \mathbf{B}_{j} \mathbf{Y}_{r+1}=\gamma_{j},
\end{aligned}
$$

where the matrices are

$$
\begin{aligned}
\mathbf{I}_{j} & =\sum_{k=0}^{m-1} \tau_{j k} \mathbf{P}(c) \mathbf{N}^{k} \\
\mathbf{B}_{j} & =\sum_{k=0}^{m-1}\left[\alpha_{j k} \mathbf{P}(a) \mathbf{N}^{k}+\beta_{j k} \mathbf{P}(b) \mathbf{N}^{k}\right] .
\end{aligned}
$$

Besides, (29) can be denoted by the augmented matrices $\left[\mathbf{I}_{j} ; \mu_{j}\right]$ and $\left[\mathbf{B}_{j} ; \gamma_{j}\right]$.

Step 4. To obtain the solution of nonlinear FVIDE (1) under the given conditions, we insert the elements of the row matrices $\left[\mathbf{I}_{j} ; \mu_{j}\right]$ or $\left[\mathbf{B}_{j} ; \gamma_{j}\right]$ to the end of the augmented matrix $\left[\mathbf{W}_{r} ; \mathbf{H}_{r}\right]$. In this way, we have the new augmented matrix $\left[\widetilde{\mathbf{W}}_{r} ; \widetilde{\mathbf{H}}_{r}\right]$, that is, $(n+m+1) \times(n+1)$ rectangular matrix.

Step 5. If $\operatorname{rank}\left(\widetilde{\mathbf{W}}_{r}\right)=\operatorname{rank}\left[\widetilde{\mathbf{W}}_{r} ; \widetilde{\mathbf{H}}_{r}\right]=n+1$, then unknown coefficients $y_{r+1}$ are uniquely determined for each iteration $r$. This kind of systems can be solved by the Gauss Elimination, Generalized Inverse, and QR factorization methods.

\section{Numerical Results}

Four numerical examples are given to illustrate the applicability, accuracy, and efficiency of the proposed method. All results are computed by using an algorithm written in Matlab 7.1. Besides, in the tables, the absolute and $\mathscr{L}^{2}$-norm errors are computed numerically on the collocation points $x_{s}=$ $a+(b-a) s / n ; s=0,1, \ldots, n$, by the folowing formulas:

$$
\begin{gathered}
E_{\mathrm{abs}}=\left|y\left(x_{s}\right)-B_{n}\left(y_{r} ; x_{s}\right)\right|, \\
E_{2}=\sqrt{\int_{0}^{x_{s}}\left(y(x)-B_{n}\left(y_{r} ; x\right)\right)^{2} d x}
\end{gathered}
$$

such that $B_{n}\left(y_{r} ; x\right)$ is a Bernstein approximation for the $r$ th iteration function $y_{r}$ and $y(x)$ is an exact solution.

Example 1. Consider the nonlinear Volterra integrodifferential equation

$$
\begin{array}{r}
y^{\prime}(x)=2 x-\frac{1}{2} \sin \left(x^{4}\right)+\int_{0}^{x} x^{2} t \cos \left(x^{2} y(t)\right) d t \\
0<x, \quad t<1
\end{array}
$$

under the initial condition $y(0)=0$. Exact solution of this equation is $y(x)=x^{2}$. Here $g\left(x, y^{\prime}(x)\right)=y^{\prime}(x)-2 x+$ $(1 / 2) \sin \left(x^{4}\right)$ and $v(x, t, y(t))=x^{2} t \cos \left(x^{2} y(t)\right)$.

Let $y_{0}(x)=0$ be the first iteration function. From Theorem 3, matrix relation of the above problem can be written as

$$
\left(\mathbf{G}_{r, 1} \mathbf{P N}+\mathbf{V}_{r, 0}\right) \mathbf{Y}_{r+1}=\mathbf{H}_{r} ; \quad r=0,1, \ldots,
$$

where $\mathbf{H}_{r}=\left[h_{r}\left(x_{s}\right)\right], \mathbf{V}_{r, 0}=\left[V_{s, i}^{r, 0}\right], \mathbf{P}=\left[p_{i, n}\left(x_{s}\right)\right]$, and $\mathbf{G}_{r, 1}=\mathbf{I}_{n+1}\left(\mathbf{I}_{n+1}\right.$ is an identity matrix with dimensional 
TABLE 1: Comparison of the $\mathscr{L}^{2}$-norm errors for Example 1.

\begin{tabular}{|c|c|c|c|c|c|c|}
\hline \multirow{3}{*}{$n$} & \multicolumn{4}{|c|}{ Presented method } & \multicolumn{2}{|c|}{ Hybrid method [29] } \\
\hline & \multicolumn{2}{|c|}{$y_{0}(x)=0$} & \multicolumn{2}{|c|}{$y_{0}(x)=x$} & & \\
\hline & $r=2$ & $r=3$ & $r=2$ & $r=3$ & $n=9$ & $8.8 e-012$ \\
\hline 2 & $7.0 e-006$ & $1.7 e-012$ & $8.7 e-008$ & $3.5 e-017$ & $n=17$ & $3.4 e-012$ \\
\hline 3 & $2.2 e-007$ & $3.8 e-017$ & $2.2 e-009$ & $7.2 e-017$ & $n=19$ & $2.1 e-013$ \\
\hline 4 & $6.0 e-008$ & $1.3 e-016$ & $1.1 e-009$ & $2.4 e-017$ & $n=39$ & $9.7 e-017$ \\
\hline
\end{tabular}

TABlE 2: Comparison of the absolute errors for Example 2.

\begin{tabular}{lcccccc}
\hline & \multicolumn{3}{c}{ Presented method } & Direct method [31] & \multicolumn{2}{c}{ Fixed point method [30] } \\
& $n=2, r=1$ & $n=2, r=2$ & $n=2, r=3$ & $n=2, r=4$ & $n=15$ & $2.9 e-005$ \\
\hline 0.1 & $5.7 e-004$ & $1.8 e-008$ & $2.5 e-017$ & $6.1 e-018$ & $9.8 e-004$ & $7.0 e-004$ \\
0.2 & $8.7 e-003$ & $2.9 e-008$ & $3.5 e-017$ & $9.0 e-018$ & $7.4 e-004$ & $1.2 e-005$ \\
0.3 & $1.0 e-003$ & $3.6 e-008$ & $4.2 e-017$ & $1.1 e-017$ & $1.1 e-003$ & $9.0 e-005$ \\
0.4 & $1.1 e-003$ & $4.0 e-008$ & $4.6 e-017$ & $1.2 e-017$ & $1.6 e-004$ & $3.1 e-004$ \\
0.5 & $9.3 e-004$ & $4.1 e-008$ & $4.7 e-017$ & $1.2 e-017$ & $1.1 e-003$ & $1.1 e-004$ \\
0.6 & $6.7 e-004$ & $3.8 e-008$ & $4.4 e-017$ & $1.2 e-017$ & $8.2 e-004$ & $1.0 e-003$ \\
0.7 & $2.5 e-004$ & $3.2 e-008$ & $3.8 e-017$ & $1.0 e-017$ & $8.2 e-004$ & $2.0 e-003$ \\
0.8 & $3.0 e-004$ & $2.2 e-008$ & $2.9 e-017$ & $7.6 e-018$ & $1.1 e-003$ & $2.9 e-003$ \\
0.9 & $1.0 e-003$ & $8.4 e-009$ & $1.6 e-017$ & $4.2 e-018$ & $1.5 e-004$ & $1.1 e-003$ \\
1.0 & $1.8 e-003$ & $8.4 e-009$ & 0 & 0 & $1.8 e-003$ \\
\hline
\end{tabular}

$(n+1))$, because of the $g_{y_{r}^{\prime}}=1$. Elements of the matrices become

$$
\begin{aligned}
& h_{r}\left(x_{s}\right) \\
& =2 x_{s}-\frac{1}{2} \sin \left(x_{s}^{4}\right) \\
& +\int_{0}^{x_{s}}\left[x_{s}^{2} t \cos \left(x_{s}^{2} y_{r}(t)\right)+x_{s}^{4} t \sin \left(x_{s}^{2} y_{r}(t)\right) y_{r}(t)\right] d t \\
& V_{s, i}^{r, 0}=\int_{0}^{x_{s}} x_{s}^{4} t \sin \left(x_{s}^{2} y_{r}(t)\right) p_{i, n}(t) d t
\end{aligned}
$$

A numerical comparison of the proposed method with the Hybrid method [29] is given in Table 1. The obtained numerical results for two different initial approximations are also listed in Table 1. It can be seen that the computational results of the proposed method are better and more effective for smaller values $n$ and iterations $r$ than the other method, and the choice of the higher degree polynomial for the initial approximation leads to better results.

Example 2. Consider the nonlinear Fredholm-Volterra integrodifferential equation

$$
\begin{aligned}
y^{\prime}(x)= & -y(x)+2 x+x^{2}+\frac{x^{5}}{10}-\frac{1}{32} \\
& +\frac{1}{4} \int_{0}^{1} t y^{3}(t) d t-\frac{1}{2} \int_{0}^{x} y^{2}(t) d t ; \quad x, t \in[0,1]
\end{aligned}
$$

with the initial condition $y(0)=0$ that exact solution is $y(x)=x^{2}$. Here the required functions and constants are denoted by $g\left(x, y(x), y^{\prime}(x)\right)=y^{\prime}(x)+y(x)-2 x-x^{2}-$ $\left(x^{5} / 10\right)+(1 / 32), f(x, t, y(t))=t y^{3}(t), v(x, t, y(t))=y^{2}(t)$, $\lambda_{1}=1 / 4$, and $\lambda_{2}=-1 / 2$.

We have two choices satisfying the initial condition for the first iteration functions such that $y_{0}(x)=0$ and $y_{0}(x)=x$. Let the first iteration function be $y_{0}(x)=x$, because of the higher degree. From Theorem 3, matrix relation of the above problem is

$$
\begin{array}{r}
\left(\mathbf{G}_{r, 0} \mathbf{P}+\mathbf{G}_{r, 1} \mathbf{P N}-\frac{1}{4} \mathbf{F}_{r, 0}+\frac{1}{2} \mathbf{V}_{r, 0}\right) \mathbf{Y}_{r+1}=\mathbf{H}_{r} ; \\
r=0,1, \ldots,
\end{array}
$$

where $\mathbf{H}_{r}=\left[h_{r}\left(x_{s}\right)\right], \mathbf{F}_{r, 0}=\left[F_{s, i}^{r, 0}\right], \mathbf{V}_{r, 0}=\left[V_{s, i}^{r, 0}\right], \mathbf{P}=$ $\left[p_{i, n}\left(x_{s}\right)\right]$, and $\mathbf{G}_{r, 0}=\mathbf{G}_{r, 1}=\mathbf{I}_{n+1}$ because of the $g_{y_{r}} \stackrel{=}{=} g_{y_{r}^{\prime}}=1$. Elements of these matrices are as follows:

$$
\begin{gathered}
F_{s, i}^{r, 0}=\int_{0}^{1} 3 t y_{r}^{2}(t) p_{i, n}(t) d t, \\
V_{s, i}^{r, 0}=\int_{0}^{x_{s}} 2 y_{r}(t) p_{i, n}(t) d t, \\
h_{r}\left(x_{s}\right)=2 x_{s}+x_{s}^{2}+\frac{x_{s}^{5}}{10}-\frac{1}{32}+\frac{1}{4} \\
\times \int_{0}^{1}\left[-2 t y_{r}^{3}(t)\right] d t-\frac{1}{2} \int_{0}^{x_{s}}\left[-y_{r}^{2}(t)\right] d t .
\end{gathered}
$$

Table 2 contains a numerical comparison of the proposed method between the numerical method based on fixed point theorem [30] and direct method by using triangular functions [31]. The table reveals that convergence of the presented method is faster and more accurate than the others. 
TABle 3: Absolute errors for Example 3.

\begin{tabular}{|c|c|c|c|c|c|c|}
\hline \multirow{2}{*}{$x$} & \multicolumn{3}{|c|}{$n=6$} & \multicolumn{3}{|c|}{$n=8$} \\
\hline & $r=1$ & $r=3$ & $r=5$ & $r=1$ & $r=3$ & $r=5$ \\
\hline 0.01 & $2.6 e-004$ & $2.6 e-004$ & $2.6 e-004$ & $5.1 e-009$ & $1.4 e-008$ & $1.4 e-008$ \\
\hline 0.02 & $2.5 e-004$ & $2.6 e-004$ & $2.6 e-004$ & $2.2 e-008$ & $1.4 e-008$ & $1.4 e-008$ \\
\hline 0.03 & $2.5 e-004$ & $2.5 e-004$ & $2.5 e-004$ & $6.6 e-008$ & $1.4 e-008$ & $1.4 e-008$ \\
\hline 0.04 & $2.5 e-004$ & $2.5 e-004$ & $2.5 e-004$ & $1.3 e-007$ & $1.4 e-008$ & $1.4 e-008$ \\
\hline 0.05 & $2.4 e-004$ & $2.5 e-004$ & $2.5 e-004$ & $2.0 e-007$ & $1.4 e-008$ & $1.4 e-008$ \\
\hline 0.06 & $2.4 e-004$ & $2.4 e-004$ & $2.4 e-004$ & $3.0 e-007$ & $1.4 e-008$ & $1.4 e-008$ \\
\hline 0.07 & $2.4 e-004$ & $2.4 e-004$ & $2.4 e-004$ & $4.1 e-007$ & $1.4 e-008$ & $1.4 e-008$ \\
\hline 0.08 & $2.3 e-004$ & $2.4 e-004$ & $2.4 e-004$ & $5.3 e-007$ & $1.4 e-008$ & $1.4 e-008$ \\
\hline 0.09 & $2.3 e-004$ & $2.3 e-004$ & $2.3 e-004$ & $6.7 e-007$ & $1.4 e-008$ & $1.4 e-008$ \\
\hline 0.1 & $2.3 e-004$ & $2.3 e-004$ & $2.3 e-004$ & $8.3 e-007$ & $1.3 e-008$ & $1.3 e-008$ \\
\hline \multirow{2}{*}{$x$} & \multicolumn{3}{|c|}{$n=10$} & \multicolumn{3}{|c|}{$n=12$} \\
\hline & $r=1$ & $r=3$ & $r=5$ & $r=1$ & $r=3$ & $r=5$ \\
\hline 0.01 & $9.1 e-009$ & $8.4 e-014$ & $8.4 e-014$ & $9.1 e-009$ & $1.1 e-016$ & $1.9 e-016$ \\
\hline 0.02 & $3.6 e-008$ & $1.2 e-013$ & $1.2 e-013$ & $3.6 e-008$ & $1.9 e-016$ & $9.6 e-017$ \\
\hline 0.03 & $8.0 e-008$ & $3.0 e-013$ & $3.0 e-013$ & $8.0 e-008$ & $2.5 e-016$ & $7.0 e-016$ \\
\hline 0.04 & $1.4 e-007$ & $3.4 e-013$ & $3.4 e-013$ & $1.4 e-007$ & $5.4 e-016$ & $4.2 e-016$ \\
\hline 0.05 & $2.2 e-007$ & $1.3 e-013$ & $1.3 e-013$ & $2.2 e-007$ & $1.9 e-015$ & $1.5 e-015$ \\
\hline 0.06 & $3.1 e-007$ & $4.7 e-013$ & $4.7 e-013$ & $3.1 e-007$ & $4.1 e-015$ & $3.7 e-015$ \\
\hline 0.07 & $4.2 e-007$ & $1.5 e-012$ & $1.5 e-012$ & $4.2 e-007$ & $7.5 e-015$ & $7.0 e-015$ \\
\hline 0.08 & $5.5 e-007$ & $3.2 e-012$ & $3.2 e-012$ & $5.5 e-007$ & $1.2 e-014$ & $1.2 e-014$ \\
\hline 0.09 & $6.9 e-007$ & $5.6 e-012$ & $5.6 e-012$ & $6.9 e-007$ & $1.9 e-014$ & $1.8 e-014$ \\
\hline 0.1 & $8.4 e-007$ & $8.8 e-012$ & $8.8 e-012$ & $8.4 e-007$ & $2.7 e-014$ & $2.6 e-014$ \\
\hline
\end{tabular}

Example 3. Consider the third-order nonlinear Fredholm integrodifferential equation

$$
y^{\prime \prime \prime}(x)=-e^{x}+\int_{-1}^{1} e^{x-2 t} y^{2}(t) d t
$$

with the boundary conditions

$$
y(0)=y^{\prime}(0)=1, \quad y(1)=e .
$$

The exact solution of the above equation is $y(x)=e^{x}$. Here $g\left(x, y^{\prime \prime \prime}(x)\right)=y^{\prime \prime \prime}(x)+e^{x}$ and $f(x, t, y(t))=e^{x-2 t} y^{2}(t)$.

Let the first iteration function be $y_{0}(x)=1+x+(e-2) x^{2}$. From Theorem 3, matrix relation of the above problem can be denoted by

$$
\left(\mathbf{G}_{r, 3} \mathbf{P N}^{3}-\mathbf{F}_{r, 0}\right) \mathbf{Y}_{r+1}=\mathbf{H}_{r} ; \quad r=0,1, \ldots
$$

such that $\mathbf{H}_{r}=\left[h_{r}\left(x_{s}\right)\right], \mathbf{F}_{r, 0}=\left[F_{s, i}^{r, 0}\right], \mathbf{P}=\left[p_{i, n}\left(x_{s}\right)\right]$, and $\mathbf{G}_{r, 3}=\mathbf{I}_{n+1}$. Here elements of matrices are, respectively,

$$
\begin{gathered}
F_{s, i}^{r, 0}=\int_{-1}^{1} 2 e^{x_{s}-2 t} y_{r}(t) p_{i, n}(t) d t, \\
h_{r}\left(x_{s}\right)=-e^{x_{s}}-\int_{-1}^{1} e^{x_{s}-2 t} y_{r}^{2}(t) d t .
\end{gathered}
$$

In Table 3, absolute errors of the proposed method are given for different values $n$ and iterations $r$. The table shows that the presented method converges quite rapidly for increasing values $n$ and iterations $r$. Besides, the absolute error of the homotopy analysis method [32] given with figure is approximately $2.0 e-006$ for iteration $r=6$. Therefore, we can say that the proposed method has more effective numerical results than the other methods.

Example 4. Consider the fourth-order Volterra integrodifferential equation

$$
y^{(4)}(x)=1+\int_{0}^{x} e^{-t} y^{2}(t) d t
$$

with the boundary conditions

$$
y(0)=y^{\prime}(0)=1, \quad y(1)=e, \quad y^{\prime}(1)=e
$$

that exact solution is $y(x)=e^{x}$. Here the functions are $g\left(x, y^{(4)}(x)\right)=y^{(4)}(x)-1$ and $v(x, t, y(t))=e^{-t} y^{2}(t)$.

Let the first iteration function be $y_{0}(x)=1+x+(2 e-$ $5) x^{2}+(3-e) x^{3}$. From Theorem 3 , matrix form of the above problem can be written as

$$
\left(\mathbf{G}_{r, 4} \mathbf{P N}^{4}-\mathbf{V}_{r, 0}\right) \mathbf{Y}_{r+1}=\mathbf{H}_{r} ; \quad r=0,1, \ldots,
$$

where $\mathbf{H}_{r}=\left[h_{r}\left(x_{s}\right)\right], \mathbf{V}_{r, 0}=\left[V_{s, i}^{r, 0}\right], \mathbf{P}=\left[p_{i, n}\left(x_{s}\right)\right]$, and $\mathbf{G}_{r, 4}=$ $\mathbf{I}_{n+1}$. Here elements of matrices are, respectively,

$$
\begin{gathered}
V_{s, i}^{r, 0}=\int_{0}^{x_{s}} 2 e^{-t} y_{r}(t) p_{i, n}(t) d t, \\
h_{r}\left(x_{s}\right)=1-\int_{0}^{x_{s}} e^{-t} y_{r}^{2}(t) d t .
\end{gathered}
$$


TABLE 4: Comparison of the $\mathscr{L}^{2}$-norm errors for Example 4.

\begin{tabular}{lccccccc}
\hline & \multicolumn{3}{c}{ Presented method } & \multicolumn{3}{c}{ Hybrid method [29] } \\
$n$ & $r=0$ & $r=1$ & $r=2$ & $r=3$ & $r=4$ & $n=15$ & $4.7 e-008$ \\
\hline 6 & $2.0 e-004$ & $2.1 e-004$ & $2.1 e-004$ & $2.1 e-004$ & $2.1 e-004$ & $n=17$ & $3.0 e-010$ \\
8 & $1.3 e-008$ & $8.3 e-009$ & $8.3 e-009$ & $8.3 e-009$ & $8.3 e-009$ & $n=19$ & $3.7 e-011$ \\
10 & $1.3 e-008$ & $1.2 e-012$ & $1.2 e-012$ & $1.2 e-012$ & $1.2 e-012$ & $n=31$ & $3.0 e-009$ \\
12 & $1.4 e-008$ & $5.4 e-015$ & $2.0 e-015$ & $2.0 e-015$ & $2.0 e-015$ & $n=35$ & $4.5 e-012$ \\
15 & $1.6 e-008$ & $5.5 e-015$ & $1.3 e-014$ & $1.3 e-014$ & $1.3 e-014$ & $n=39$ & $6.0 e-013$ \\
\hline
\end{tabular}

In Table $4, \mathscr{L}^{2}$-norm errors of the proposed method are compared with the $\mathscr{L}^{2}$-norm errors computed via the Hybrid of Block-Pulse functions and Lagrange interpolation polynomials [29] for different values $n$. While the results of other methods are effective, the computational results of the proposed method are more rapid, effective, for smaller values $n$.

\section{Conclusions}

In general, nonlinear integrodifferential equations can not be solved analytically. For this reason, numerical solutions of nonlinear equations are needed. With the presented method, we have reduced the nonlinear FVIDE (1) to a sequence of linear equations depending on the collocation points and the iteration function, and then, combining the conditions with obtained linear matrix equation, we have the generalized Bernstein polynomials solution. Besides, the proposed method is valid for both nonlinear differential and integral equations; this is explained in Section 2. If unknown function is continuous on the interval $[a, b]$, then the Bernstein collocation method can be used for solving these kinds of equations via the quasilinearization technique. For convenience, the first iteration function can be choosen as initial value. Besides, this function can be select as the higher degree polynomials satisfied the given conditions for providing the better approximation. These polynomials can be obtained from interpolation or the least square approximation methods. The applicability and accuracy of the proposed method have been tested with some numerical examples. These results have shown that the presented method converges rapidly for all problems. Consequently, all these positive implications lead to applicability of the proposed method for numerical solutions of any other kinds of problems including nonlinear equations.

\section{Conflict of Interests}

The authors declare that there is no conflict of interests regarding the publication of this paper.

\section{Acknowledgment}

This work is supported by Scientific Research Project Coordination Unit of Pamukkale University with no. 2012FBE036.

\section{References}

[1] R. E. Bellman and R. E. Kalaba, Quasilinearization and Nonlinear Boundary Value Problems, Elsevier, New York, NY. USA, 1965.

[2] E. L. Stanley, Quasilinearization and Invariant Imbedding, Academic Press, New York, NY, USA, 1968.

[3] R. P. Agarwal and Y. M. Chow, "Iterative methods for a fourth order boundary value problem," Journal of Computational and Applied Mathematics, vol. 10, no. 2, pp. 203-217, 1984.

[4] A. Akyuz Dascioglu and N. Isler, "Bernstein collocation method for solving nonlinear differential equations," Mathematical \& Computational Applications, vol. 18, no. 3, pp. 293-300, 2013.

[5] A. Charles and J. Baird, "Modified quasilinearization technique for the solution of boundary-value problems for ordinary differential equations," Journal of Optimization Theory and Applications, vol. 3, no. 4, pp. 227-242, 1969.

[6] V. B. Mandelzweig and F. Tabakin, "Quasilinearization approach to nonlinear problems in physics with application to nonlinear ODEs," Computer Physics Communications, vol. 141, no. 2, pp. 268-281, 2001.

[7] J. I. Ramos, "Piecewise quasilinearization techniques for singular boundary-value problems," Computer Physics Communications, vol. 158, no. 1, pp. 12-25, 2004.

[8] B. Ahmad, R. Ali Khan, and S. Sivasundaram, "Generalized quasilinearization method for nonlinear functional differential equations," Journal of Applied Mathematics and Stochastic Analysis, vol. 16, no. 1, pp. 33-43, 2003.

[9] Z. Drici, F. A. McRae, and J. V. Devi, "Quasilinearization for functional differential equations with retardation and anticipation," Nonlinear Analysis: Theory, Methods \& Applications, vol. 70, no. 4, pp. 1763-1775, 2009.

[10] F. Caliò, F. Munoz, and E. Marchetti, "Direct and iterative methods for the numerical solution of mixed integral equations," Applied Mathematics and Computation, vol. 216, no. 12, pp. 3739-3746, 2010.

[11] K. Maleknejad and E. Najafi, "Numerical solution of nonlinear Volterra integral equations using the idea of quasilinearization," Communications in Nonlinear Science and Numerical Simulation, vol. 16, no. 1, pp. 93-100, 2011.

[12] S. G. Pandit, "Quadratically converging iterative schemes for nonlinear volterra integral equations and an application," Journal of Applied Mathematics and Stochastic Analysis, vol. 10, no. 2, pp. 169-178, 1997.

[13] B. Ahmad, "A quasilinearization method for a class of integrodifferential equations with mixed nonlinearities," Nonlinear Analysis: Real World Applications, vol. 7, no. 5, pp. 997-1004, 2006. 
[14] B. Ahmad, R. A. Khan, and S. Sivasundaram, "Generalized quasilinearization method for integro-differential equations," Nonlinear Studies, vol. 8, no. 3, pp. 331-341, 2001.

[15] P. Wang, Y. Wu, and B. Wiwatanapaphee, "An extension of method of quasilinearization for integro-differential equations," International Journal of Pure and Applied Mathematics, vol. 54, no. 1, pp. 27-37, 2009.

[16] R. T. Farouki and V. T. Rajan, "Algorithms for polynomials in Bernstein form," Computer Aided Geometric Design, vol. 5, no. 1, pp. 1-26, 1988.

[17] G. G. Lorentz, Bernstein Polynomials, Chelsea, New York, NY, USA, 2nd edition, 1986.

[18] E. H. Doha, A. H. Bhrawy, and M. A. Saker, "Integrals of Bernstein polynomials: an application for the solution of high even-order differential equations," Applied Mathematics Letters, vol. 24, no. 4, pp. 559-565, 2011.

[19] O. R. Işık, M. Sezer, and Z. Güney, "A rational approximation based on Bernstein polynomials for high order initial and boundary values problems," Applied Mathematics and Computation, vol. 217, no. 22, pp. 9438-9450, 2011.

[20] S. Bhattacharya and B. N. Mandal, "Use of Bernstein polynomials in numerical solutions of Volterra integral equations," Applied Mathematical Sciences, vol. 2, no. 33-36, pp. 1773-1787, 2008.

[21] K. Maleknejad, E. Hashemizadeh, and R. Ezzati, "A new approach to the numerical solution of Volterra integral equations by using Bernstein's approximation," Communications in Nonlinear Science and Numerical Simulation, vol. 16, no. 2, pp. 647-655, 2011.

[22] B. N. Mandal and S. Bhattacharya, "Numerical solution of some classes of integral equations using Bernstein polynomials," Applied Mathematics and Computation, vol. 190, no. 2, pp. 17071716, 2007.

[23] A. Shirin and M. S. Islam, "Numerical solutions of Fredholm integral equations using Bernstein polynomials," Journal of Scientific Research, vol. 2, no. 2, pp. 264-272, 2010.

[24] V. K. Singh, R. K. Pandey, and O. P. Singh, "New stable numerical solutions of singular integral equations of Abel type by using normalized Bernstein polynomials," Applied Mathematical Sciences, vol. 3, no. 5-8, pp. 241-255, 2009.

[25] O. R. Işık, M. Sezer, and Z. Güney, "Bernstein series solution of a class of linear integro-differential equations with weakly singular kernel," Applied Mathematics and Computation, vol. 217, no. 16, pp. 7009-7020, 2011.

[26] Y. Ordokhani and S. Davaei far, "Application of the Bernstein polynomials for solving the nonlinear Fredholm integrodifferential equations," Journal of Applied Mathematics \& Bioinformatics, vol. 1, no. 2, pp. 13-31, 2011.

[27] B. M. Pandya and D. C. Joshi, "Solution of a Volterra's population model in a Bernstein polynomial basis," Applied Mathematical Sciences, vol. 5, no. 69, pp. 3403-3410, 2011.

[28] G. M. Phillips, Interpolation and Approximation by Polynomials, Springer, New York, NY, USA, 2003.

[29] H. R. Marzban and S. M. Hoseini, "Solution of nonlinear Volterra-Fredholm integrodifferential equations via hybrid of block-pulse functions and Lagrange interpolating polynomials," Advances in Numerical Analysis, vol. 2012, Article ID 868279, 14 pages, 2012.

[30] M. I. Berenguer, D. Gámez, and A. J. López Linares, "Fixed point techniques and Schauder bases to approximate the solution of the first order nonlinear mixed Fredholm-Volterra integrodifferential equation," Journal of Computational and Applied Mathematics, vol. 252, pp. 52-61, 2013.

[31] E. Babolian, Z. Masouri, and S. Hatamzadeh-Varmazyar, "Numerical solution of nonlinear Volterra-Fredholm integrodifferential equations via direct method using triangular functions," Computers \& Mathematics with Applications, vol. 58, no. 2, pp. 239-247, 2009.

[32] A. Shidfar, A. Molabahrami, A. Babaei, and A. Yazdanian, "A series solution of the nonlinear Volterra and Fredholm integrodifferential equations," Communications in Nonlinear Science and Numerical Simulation, vol. 15, no. 2, pp. 205-215, 2010. 


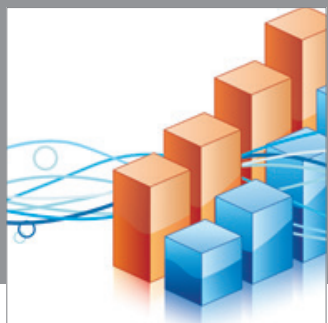

Advances in

Operations Research

mansans

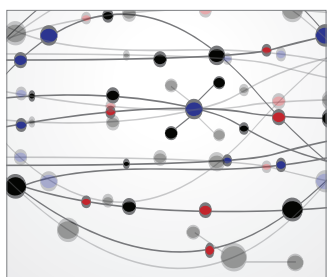

The Scientific World Journal
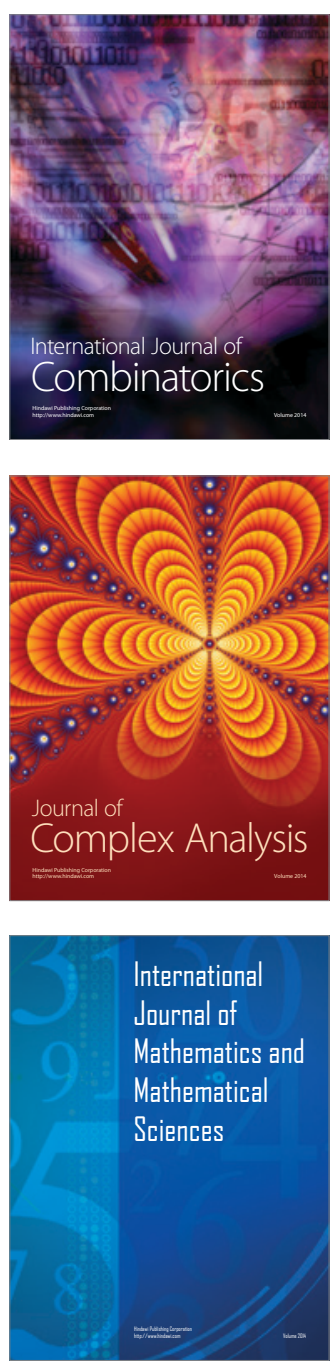
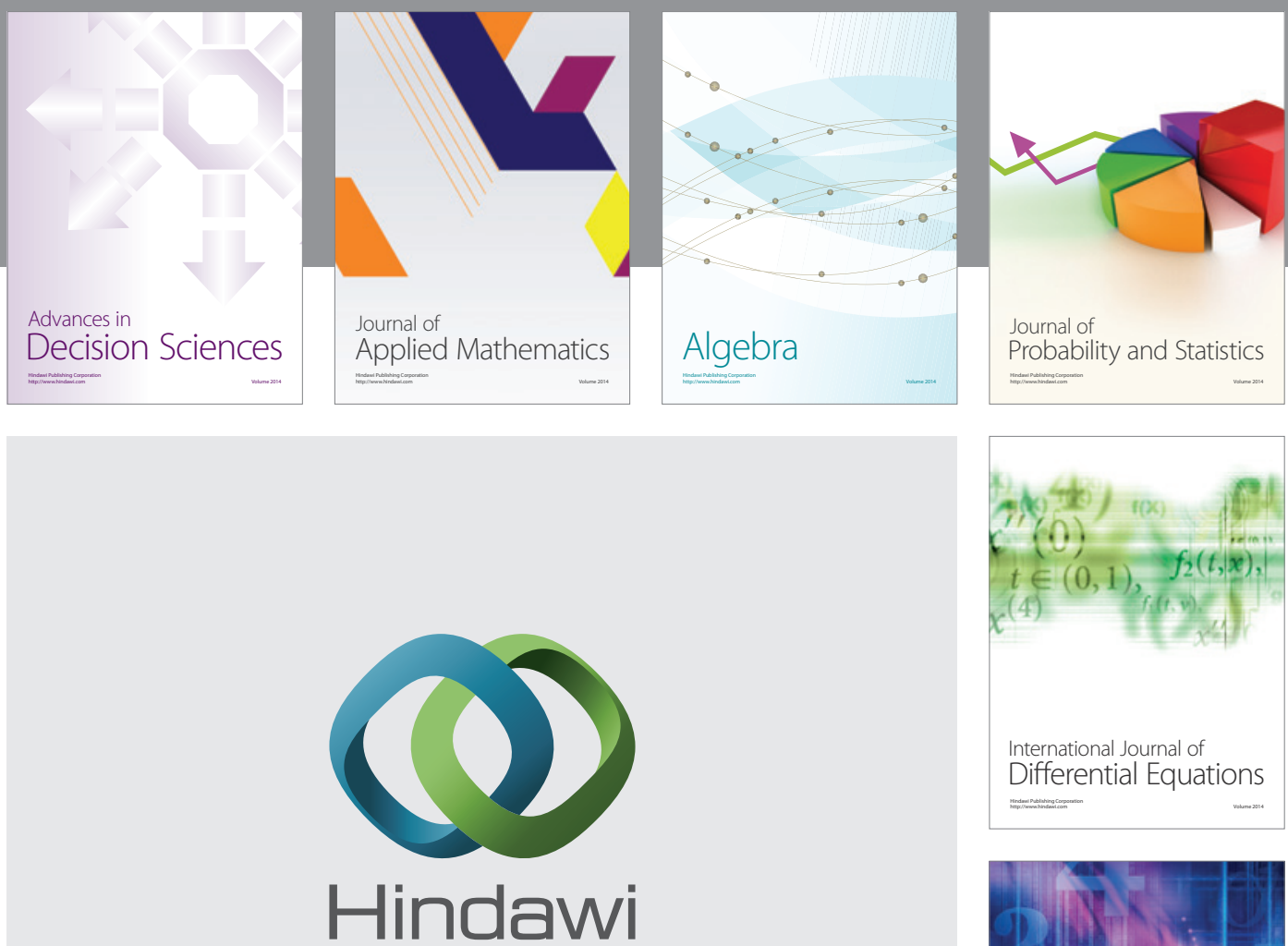

Submit your manuscripts at http://www.hindawi.com
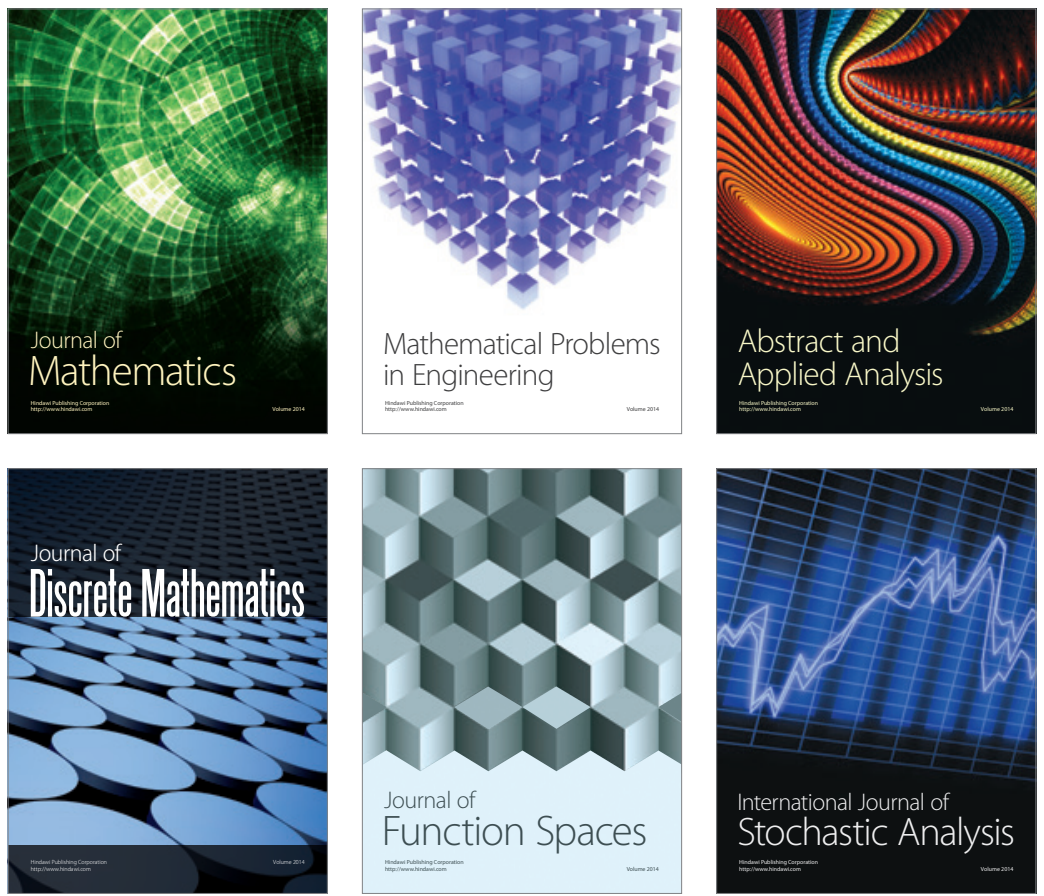

Journal of

Function Spaces

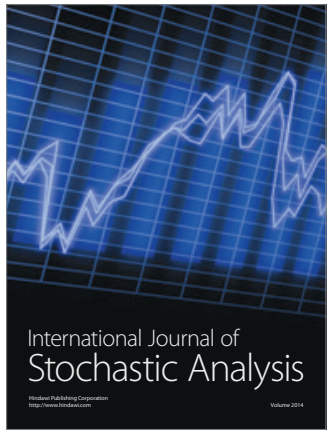

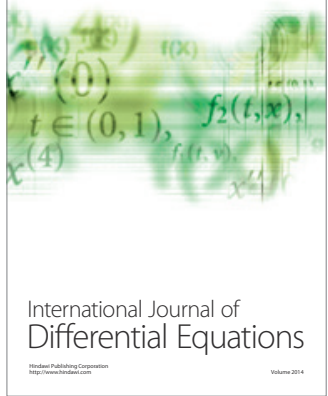
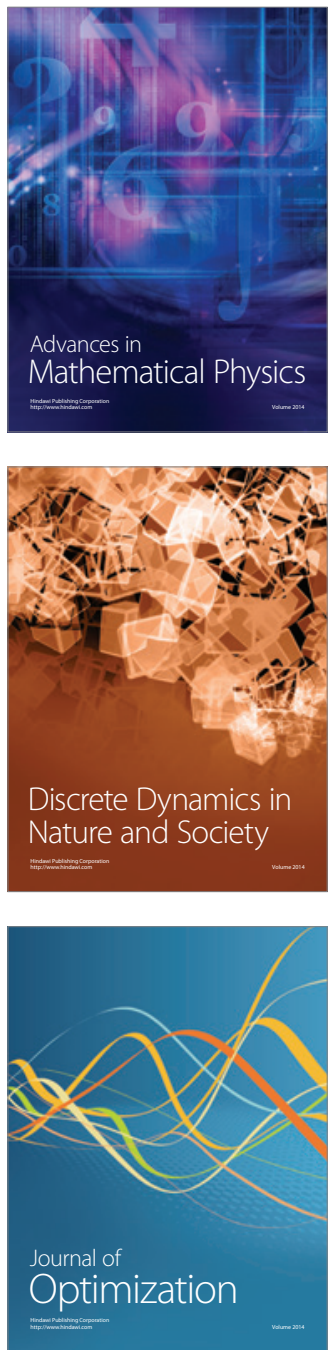